\title{
Rate of increase in serum lactate level risk-stratifies infants after surgery for congenital heart disease
}

\author{
Kurt R. Schumacher, MS, MD, Rebecca A. Reichel, BS, Jeffrey R. Vlasic, MS, Sunkyung Yu, MS, \\ Janet Donohue, MPH, Robert J. Gajarski, MD, and John R. Charpie, MD, PhD
}

\begin{abstract}
Objective: Increased blood lactate levels reflect tissue oxygen debt and might be indicative of low cardiac output. We hypothesized that the rate of increase in serum lactate would be an ideal marker to discriminate between infants at high and low risk of a poor outcome after surgical repair of congenital heart disease using cardiopulmonary bypass.
\end{abstract}

Methods: In the present prospective, observational study in a pediatric cardiac intensive care unit, infants (aged $<12$ months) undergoing cardiac surgery had serial whole blood lactate levels measured with every arterial blood gas drawn for the first 24 postoperative hours. The composite poor outcome included death, the need for extracorporeal support, and dialysis.

Results: The lactate levels were measured in 231 infants; 19 infants $(8.2 \%)$ had a poor outcome. A lactate increase rate of $0.6 \mathrm{mmol} / \mathrm{L} / \mathrm{h}$ had very good discriminatory ability (area under the curve [AUC], 0.89) with a sensitivity of $90 \%$, specificity of $84 \%$, positive predictive value (PPV) of $34 \%$, and negative predictive value (NPV) of $99 \%$. Similar results were obtained for subgroups stratified by 1- or 2-ventricle heart disease and risk adjustment for congenital heart surgery (RACHS-1) score. In neonates (age $<30$ days) with single-ventricle physiology $(\mathrm{n}=43$, poor outcome $=8)$, a lactate increase of $0.6 \mathrm{mmol} / \mathrm{L} / \mathrm{h}$ had near perfect discriminatory ability (AUC 0.99 ) with a sensitivity of $100 \%$, specificity of $51 \%$, PPV of $32 \%$, and NPV of $100 \%$. In 2-ventricle neonates $(\mathrm{n}=43$, poor outcome $=5)$, a lactate increase of $0.6 \mathrm{mmol} / \mathrm{L} / \mathrm{h}$ also had near perfect discriminatory ability (AUC, 0.99 ), with a sensitivity of $100 \%$, specificity of $90 \%$, PPV of $56 \%$, and NPV of $100 \%$.

Conclusions: The postoperative lactate increase rate allows discrimination between infants at high and low risk of morbidity and mortality after congenital heart disease surgery, and the lactate level can be followed serially for the treatment response. (J Thorac Cardiovasc Surg 2014;148:589-95)

Clinical indicators reflecting tissue perfusion and systemic oxygen delivery are needed after surgery for congenital heart disease (CHD). The ideal indicator would have a high predictive value for outcome, would allow for early detection of tissue ischemia before injury, and could be monitored serially to assess the response to therapeutic interventions. Blood lactate has been studied as a potential clinical marker, particularly in children after cardiac surgery. Lactate is formed from pyruvate as the final product of glycolysis. Under aerobic conditions, lactate is converted back to pyruvate by mitochondrial oxidation. Under anaerobic conditions, cellular lactate levels increase, leading to increased serum lactate levels. ${ }^{1}$ Thus, hyperlactemia might reflect tissue oxygen debt.

\footnotetext{
From the University of Michigan Congenital Heart Center, C. S. Mott Children's Hospital, Ann Arbor, Mich.

Disclosures: Authors have nothing to disclose with regard to commercial support. Received for publication April 21, 2013; revisions received Aug 13, 2013; accepted for publication Sept 16, 2013; available ahead of print Oct 21, 2013.

Address for reprints: Kurt R. Schumacher, MS, MD, University of Michigan Congenital Heart Center, C.S. Mott Children's Hospital, 1540 E Hospital Dr, Ann Arbor, MI 48109 (E-mail: kurts@med.umich.edu). $0022-5223 / \$ 36.00$

Copyright (c) 2014 by The American Association for Thoracic Surgery http://dx.doi.org/10.1016/j.jtcvs.2013.09.002
}

Multiple studies in the pediatric data have examined the utility of blood lactate levels as both positive and negative predictors of outcome after surgery for CHD. ${ }^{2-10}$ However, most of these studies had important limitations, including low patient numbers, that compromised their ability to adequately assess the predictive value of an elevated lactate level. Furthermore, several studies combined data from children of all ages in their analyses. This could have been problematic because the susceptibility to tissue oxygen debt and normal lactate levels vary with age. ${ }^{11,12}$ Finally, the point at which the lactate level is measured during the clinical course and the chronicity of the lactate measurements differed from study-to-study, making comparisons among smaller studies difficult.

More than 1 decade ago, we published a prospective study on the predictive value of serial lactate levels in a small cohort of 46 neonates after complex congenital heart surgery at the University of Michigan. ${ }^{13}$ In that limited sample, the rate of increase in serum lactate was identified as an early marker of increasing tissue oxygen debt that was predictive of poor outcome. Despite the potential benefits of an increase in lactate as a predictor of poor outcome, these results have not been reproduced on a larger 


$$
\begin{aligned}
& \text { Abbreviations and Acronyms } \\
& \begin{aligned}
\text { AUC } & =\text { area under the curve } \\
\text { CBP } & =\text { cardiopulmonary bypass } \\
\text { CHD } & =\text { congenital heart disease } \\
\text { CICU } & =\text { cardiac intensive care unit } \\
\text { ECMO } & =\text { extracorporeal membrane oxygenation } \\
\text { ICU } & =\text { intensive care unit } \\
\text { IQR } & =\text { interquartile range } \\
\text { NPV } & =\text { negative predictive value } \\
\text { PPV } & =\text { positive predictive value } \\
\text { RACHS-1 } & =\text { risk adjustment for congenital heart } \\
& \text { surgery }
\end{aligned}
\end{aligned}
$$

scale or in a broader patient population. In the present study, we sought to re-evaluate the utility of both initial and serial lactate measurements in a larger cohort of infants after cardiopulmonary bypass (CPB) for congenital heart surgery. We hypothesized that rate of increase in serum lactate would have a strong predictive value for the outcome and would proceed maximal tissue oxygen debt.

\section{METHODS \\ Patients}

We conducted a prospective, observational study of infants $(<12$ months of age) after surgical repair of CHD requiring CPB. A total of 231 nonconsecutive infants were enrolled in the study from June 2010 to September 2011. Nonconsecutive enrollment resulted because of parental refusal (3 infants) or parental unavailability for consent (18 infants). Parental consent was obtained before data collection, and the institutional review board of the University of Michigan approved the research protocol. All infants were required to be $\geq 37$ weeks' corrected gestational age and to weigh $>2 \mathrm{~kg}$ before enrollment. Infants requiring extracorporeal membrane oxygenation (ECMO) in the operating room before arrival in the cardiac intensive care unit (CICU) were excluded from the study. Only those patients in whom ECMO was initiated emergently after postoperative CICU admission were included in the present analysis.

\section{Lactate Measurements}

Serial, arterial, whole blood lactate levels were collected per CICU protocol at the discretion of the treating clinician. At our center, lactate levels were obtained routinely with each arterial blood gas drawn. All lactate levels measured in the initial, postoperative 24 hours were included in the present study. Whole blood lactate was measured using a pediatric blood gas analyzer (ABL 800 Flex; Radiometer, Brønshøj, Denmark) for each sample. For serum lactate levels within a range of 0 to $30 \mathrm{mmol} / \mathrm{dL}$, this pediatric blood gas analyzer is accurate, with an $\mathrm{r}^{2}$ of 0.95 , and precise, with a coefficient of variance of $0 \%$ to $2 \%$ compared with laboratorymeasured lactate values. A priori analyses were planned to assess the predictive values of both the initial postoperative lactate level and the maximal rate of increase in the lactate level. The initial lactate level was drawn within 1 hour of arrival in the CICU postoperatively. The lactate increase rate was calculated as the difference between the consecutive lactate measurements divided by the interval between the measurements. The maximal rate of lactate increase was expressed as the largest increase between 2 consecutive measurements divided by interval during the first 24 postoperative hours.

\section{Outcome Variable}

The primary outcome variable ("poor outcome") was a composite of death during hospitalization, the need for ECMO support initiated after admission to the CICU, and the initiation of renal dialysis. In our CICU, few infants have significant preoperative kidney disease, and most cases of renal injury requiring dialysis result from acute kidney injury caused by tissue ischemia, which has been reported in other studies. ${ }^{14}$ Although not inclusive of all significant postoperative events that could result from postoperative ischemia, this composite outcome was specifically selected because the individual outcomes can be easily assessed as positive or negative, and the endpoints are clinically relevant. A subject was classified as having a poor outcome based on the appearance of any 1 component of the composite outcome. Secondary outcomes included the need for an unplanned cardiac reoperation, duration of tracheal intubation, and length of intensive care unit (ICU) and total hospital stays.

\section{Statistical Analysis}

Descriptive statistics are reported as frequencies with percentages for categorical variables and medians with interquartile ranges (IQRs) for continuous variables. The demographic and patient surgical characteristics between the 2 outcome groups (poor and good outcome) were compared using the chi-square test or Fisher's exact test, as appropriate, for the categorical variables and the Wilcoxon rank sum test for continuous variables. Using receiver operating characteristic curves, the optimal levels for the initial postoperative lactate level and maximal hourly increase in lactate were determined as the best combination of sensitivity and specificity for significant discrimination between patient outcomes. To ascertain the predictive ability of the optimal levels, the area under the curve (AUC), sensitivity, specificity, positive predictive value (PPV), and negative predictive value (NPV) for each optimal value were calculated, and the relative risks with the $95 \%$ confidence intervals (CIs) for a poor outcome are also reported. Similarly, additional subgroup analyses planned a priori included evaluating the predictive value of the initial lactate level and increase in lactate stratified by single- and 2-ventricle subjects, singleand 2-ventricle neonates (age $<30$ days), and risk adjustment for congenital heart surgery (RACHS-1) score. ${ }^{15}$ Finally, after stratifying the cohort by the high- and low-risk lactate measurements, other clinical outcomes were compared using the chi-square test or Fisher's exact test, as appropriate, for the categorical variables and the Wilcoxon rank sum test for continuous variables. All analyses were performed using Statistical Analysis Systems, version 9.3 (SAS Institute, Cary, NC), with statistical significance set at $P<.05$ using 2 -sided tests.

\section{RESULTS}

A total of 231 subjects were analyzed in the present study. Of these 231 subjects, 212 had good outcomes and 19 $(8.2 \%)$ met $\geq 1$ of the criteria for a composite poor outcome, including $9(3.9 \%)$ who required post-CICU admission ECMO support, 7 who required renal dialysis $(3.0 \%)$, and $11(4.8 \%)$ who died (Figure 1). The interval between surgery and the occurrence of the composite poor outcome was a median of 1 day (IQR, 0-63). Specifically, the interval from surgery to a poor outcome was a median of 1 day (range, $0-2$ ) for patients needing ECMO support, a median of 3 days (range, 2-15) for patients requiring renal dialysis, and a median of 24 days (range, 1-197) for the patients who died. Six additional subjects whose parents had agreed to the study were excluded from the analysis after requiring ECMO support in the operating room before the CICU admission. 


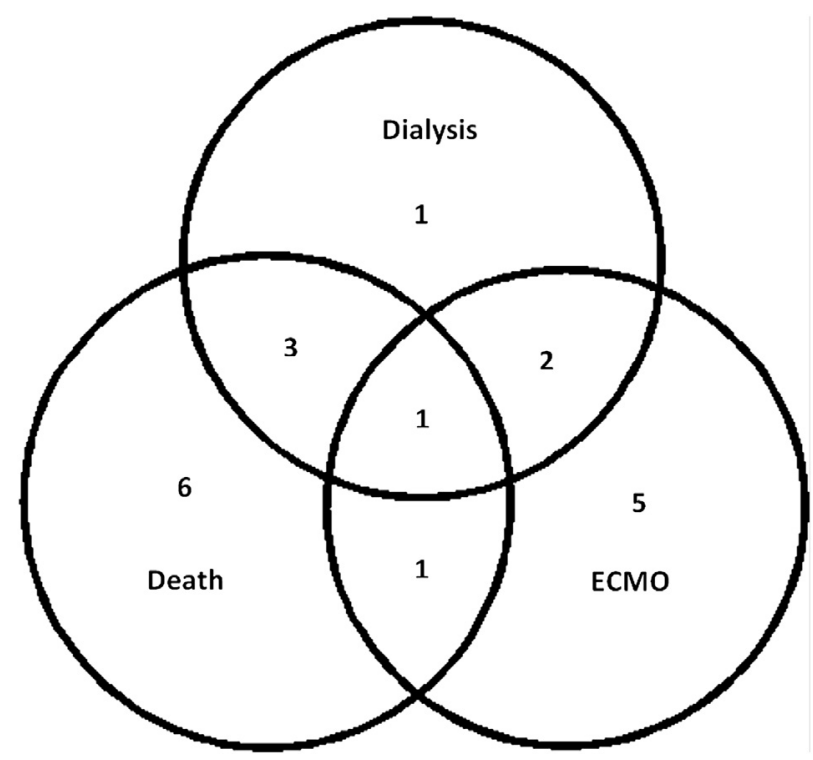

FIGURE 1. Venn diagram illustrating the distribution of poor outcomes among the 19 subjects meeting the composite poor outcome endpoint. $E C M O$, Extracorporeal membrane oxygenation.

The demographics and patient surgical data are listed in Table 1. Significant differences between the outcome groups included younger age, higher RACHS-1 scores,

TABLE 1. Demographic and clinical characteristics $(n=231)$

\begin{tabular}{lcccc}
\hline & & \multicolumn{2}{c}{ Outcome } & \\
\cline { 3 - 4 } Characteristics & All & $\begin{array}{c}\text { Poor } \\
(\mathbf{n}=\mathbf{1 9})\end{array}$ & $\begin{array}{c}\text { Good } \\
(\mathbf{n}=\mathbf{2 1 2})\end{array}$ & $\begin{array}{c}\boldsymbol{P} \\
\text { value* }\end{array}$ \\
\hline Male gender & $107(46.3)$ & $11(57.9)$ & $96(45.3)$ & .29 \\
White race & $161(69.7)$ & $12(63.2)$ & $149(70.3)$ & .75 \\
Age at surgery (d) & $82(8-148)$ & $9(5-34)$ & $92(9.5-148.5)$ & .02 \\
RACHS-1 score & & & & $.01 \dagger$ \\
1 or 2 & $89(38.5)$ & $2(10.5)$ & $87(41.0)$ & \\
3 & $59(25.5)$ & $2(10.5)$ & $57(26.9)$ & \\
4 & $29(12.6)$ & $5(26.3)$ & $24(11.3)$ & \\
5 or 6 & $39(16.9)$ & $7(36.8)$ & $32(15.1)$ & \\
$\quad$ NA & $15(6.5)$ & $3(15.8)$ & $12(5.7)$ & \\
Single- vs 2-ventricle & & & & \\
$\quad$ disease & & & & \\
Single & $69(29.9)$ & $10(52.6)$ & $59(27.8)$ & \\
$\quad$ Two & $162(70.1)$ & $9(47.4)$ & $153(72.2)$ & \\
Intraoperative & & & & \\
CPB (min) & $93(68-129)$ & $111(75-162)$ & $92.5(66.5-126)$ & .22 \\
Aortic crossclamp & $39(25-61)$ & $38(33-56)$ & $39.5(24.5-61)$ & .87 \\
$\quad$ time (min) & & & & \\
Hypothermic & $59(25.5)$ & $11(57.9)$ & $48(22.6)$ & .002 \\
$\quad$ cardiac arrest & & & & \\
\hline
\end{tabular}

$\overline{\text { Data are presented as } \mathrm{n}(\%) \text { for categorical variables and median (interquartile range) }}$ for continuous variables. RACHS-1, Risk adjustment for congenital heart surgery; $C P B$, cardiopulmonary bypass. $* P$ value from chi-square test or Fisher's exact test for categorical variables and Wilcoxon rank sum test for continuous variables for comparison of each characteristic between 2 outcome groups. †Comparison for RACHS- 1 classification was made as 1 to 4 versus 5 or 6 , with $P$ value from Fisher's exact test. more single-ventricle heart disease, and a greater percentage of patients undergoing circulatory arrest in the poor outcome group. A post hoc chart review found that no patient was given ECMO because of a lactate level alone. The lactate levels were available to the treating clinician; however, factors other than lactate were reported as the primary indication for the initiation of ECMO support in each case. The indications for ECMO were cardiopulmonary resuscitation without regaining a perfusing heart rhythm or adequate blood pressure (extracorporeal cardiopulmonary resuscitation) in 4 patients, successful cardiopulmonary resuscitation with subsequent hemodynamic status judged to be unstable in 2 patients, and inadequate hemodynamic status despite maximal, nonmechanical support in 3 patients. The indications for renal dialysis were severe edema with oliguria in 5 subjects and oliguria with electrolyte abnormalities in 2 subjects.

The median number of lactate assessments in the first postoperative 24 hours was 10 per patient (minimum 6 , maximum 25). The median initial lactate value among the poor outcome patients was $6.2 \mathrm{mmol} / \mathrm{L}$ (IQR, 2.2-7.5). The initial lactate level in the good outcome group was significantly lower at $2.0 \mathrm{mmol} / \mathrm{L}$ (IQR, 1.4-4; $P<.0001$ ). During the first 24 postoperative hours, the median peak lactate value among the poor outcome patients was 10.9 $\mathrm{mmol} / \mathrm{L}$ (IQR, 6.5-17) compared with a median peak lactate value of $2.4 \mathrm{mmol} / \mathrm{L}$ (IQR, 1.7-4.3) among the good outcome subjects $(P<.0001)$. The maximal increase in serum lactate was calculated for each patient. Among those with a poor outcome, the median peak lactate increase was $2 \mathrm{mmol} / \mathrm{L} / \mathrm{h}$ (IQR, 0.7-3.4) compared with $0.25 \mathrm{mmol} / \mathrm{L} / \mathrm{h}$ (IQR, 0.1-0.5) among those with a good outcome $(P<.0001)$. The median interval between consecutive measurements used to calculate the maximal increase in lactate was 1.2 hours (IQR, 0.8-1.5) in the poor outcome group and 2.1 hours (IQR, 1.4-3.2) in the good outcome group $(P<.001)$.

The optimal levels that discriminated between patient outcomes for both the initial postoperative lactate level and the maximal hourly lactate increase were determined using receiver operating characteristic curves (Figure 2). An optimal initial lactate level of $5 \mathrm{mmol} / \mathrm{L}$ showed good discrimination between the outcome groups, with an AUC of 0.78 (95\% CI, 0.66-0.91), a sensitivity of $68.4 \%$, and a specificity of $85.8 \%$ for a poor outcome. An optimal predictive increase in lactate of $0.6 \mathrm{mmol} / \mathrm{L} / \mathrm{h}$ demonstrated very good discriminatory ability, with an AUC of 0.89 (95\% CI, 0.78-1.00), a sensitivity of $89.5 \%$, and a specificity of $84.4 \%$ for a poor outcome. For both cutoff values, the PPV was relatively low, but the NPV was very high. Also, both the initial lactate level and the rate of increase conferred an increase in the relative risk of a poor outcome, with a greater increase in the risk of increasing lactate levels (Table 2). 

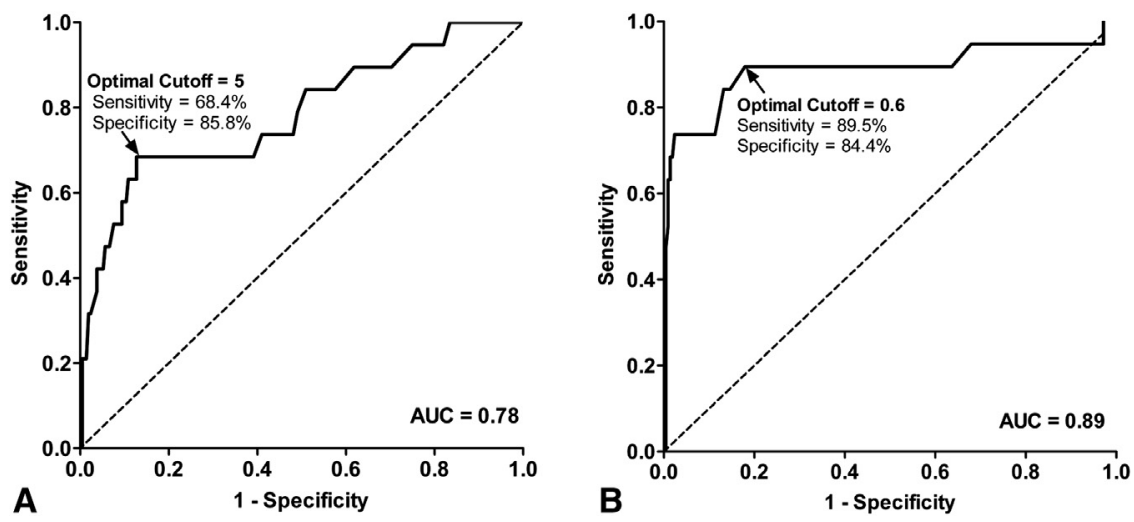

FIGURE 2. Receiver operator characteristic curves for (A) initial lactate value and (B) maximal hourly increase in lactate. AUC, Area under the curve.

A total of 50 patients had lactate levels that increased faster than $0.6 \mathrm{mmol} / \mathrm{L} / \mathrm{h}$. In 45 of 50 patients, the lactate increase was measured a median of 4.6 hours (IQR, 2.1-9.4) before reaching their peak lactate level after the initial increase. In the remaining 5 patients, the peak lactate level also constituted the end of the maximal calculated increase. Thus, in $90 \%$ of patients with an increasing lactate level, the increase preceded the maximal tissue oxygen debt, as measured by the peak lactate level that followed the lactate increase.

We examined the interaction between the initial and increasing lactate levels. For patients who had a lactate increase of $\geq 0.6 \mathrm{mmol} / \mathrm{L} / \mathrm{h}$, the initial lactate value was a median of $4.9 \mathrm{mmol} / \mathrm{L}$ (IQR, 2.6-7.0). For patients who did not have a lactate increase of $\geq 0.6 \mathrm{mmol} / \mathrm{L} / \mathrm{h}$, the median initial lactate level was $1.8 \mathrm{mmol} / \mathrm{L}$ (IQR, 1.4-3.3). The correlation between high initial lactate values and increasing lactate values among all subjects was analyzed. Of the 50 patients with a lactate increase of $\geq 0.6 \mathrm{mmol} / \mathrm{L} / \mathrm{h}, 25$ patients did not have an initial lactate of $\geq 5 \mathrm{mmol} / \mathrm{L}$. Thus, although the correlation between the 2 groups was significant $(P<.001)$, a significant proportion of patients had an increasing lactate level who had not presented with an initial high lactate level. That suggests that although some subjects have a significant tissue oxygen debt because of their intraoperative course that extends postoperatively, another, equally large, patient group will has a tissue oxygen debt that primarily evolves in the postoperative period. Among the 19 subjects with a poor outcome, $12(63.2 \%)$ had both high initial and increasing lactate levels, $5(26.3 \%)$ had increasing lactate levels but had low initial lactate levels, $1(5.2 \%)$ had a high lactate level that did not increase and $1(5.2 \%)$ had neither a high initial nor increasing lactate level. The predictive value of a combined high initial and increasing lactate was assessed. The combined lactate measure had an AUC of $0.79,63.2 \%$ sensitivity, $93.9 \%$ specificity, $48.0 \% \mathrm{PPV}$, and $96.6 \%$ NPV. Thus, the combined measure had a greater PPV than either lactate measure alone but had a discriminatory ability similar to the initial lactate level and inferior to an increasing lactate level.

As an additional analysis, we limited the primary outcome to include only emergent ECMO support and death. An initial lactate level of $5 \mathrm{mmol} / \mathrm{L}$ demonstrated good discrimination between outcomes with an AUC of 0.78 (95\% CI, 0.65-0.91), a sensitivity of $66.7 \%$, and a specificity of $85.4 \%$. An increase in the lactate level of $0.6 \mathrm{mmol} / \mathrm{L}$ demonstrated very good discrimination, with an AUC of 0.88 (95\% CI, 0.76-1.00), a sensitivity of $88.9 \%$, and a specificity of $84.0 \%$. These results are significant and similar to, but slightly less precise than, those using a composite outcome, including dialysis.

\section{Subgroup Analyses}

The diagnostic performance of the initial lactate and increase in lactate was assessed after stratifying the subjects by single- versus 2 -ventricle heart anatomy and physiology. Similar to the overall cohort, an increase in lactate of $0.6 \mathrm{mmol} / \mathrm{L} / \mathrm{h}$ again demonstrated superior discriminatory ability and had a high NPV but low PPV. In subjects with the single-ventricle physiology, an initial lactate cutoff value of $5 \mathrm{mmol} / \mathrm{L}$ had an AUC of 0.74 , PPV $30 \%$, and NPV $95 \%$. In single-ventricle subjects, a increase in the lactate cutoff of $0.6 \mathrm{mmol} / \mathrm{L} / \mathrm{h}$ had an AUC 0.87,

TABLE 2. Most predictive blood lactate measurements for a poor outcome $(n=231$, poor outcome, $n=19)$

\begin{tabular}{lccrc}
\hline \multicolumn{1}{c}{ Lactate } & Cutoff value* $(\mathbf{m m o l} / \mathbf{L})$ & PPV $(\%)$ & NPV $(\%)$ & RR $(\mathbf{9 5} \% \mathbf{C I})$ \\
\hline Initial lactate level & 5 & 30.2 & 96.8 & $9.5(3.8-23.5)$ \\
Maximal change in lactate level/h & 0.60 & 34.0 & 98.9 & $30.8(7.4-128.7)$ \\
\hline
\end{tabular}

$P P V$, Positive predictive value; $N P V$, negative predictive value; $R R$, relative risk; $C I$, confidence interval. *The optimal cutoff value was obtained from generating receiver operating characteristic curves. 
TABLE 3. Most predictive blood lactate concentration for poor outcome stratified by single- or 2-ventricle repair in neonates (n = 86)

\begin{tabular}{|c|c|c|c|c|c|c|}
\hline Lactate level & Cutoff value (mmol/L) & AUC & Sensitivity $(\%)$ & Specificity $(\%)$ & PPV $(\%)$ & NPV (\%) \\
\hline \multicolumn{7}{|l|}{ All neonates } \\
\hline Initial lactate level & 5 & 0.69 & 76.9 & 61.6 & 26.3 & 93.7 \\
\hline Maximal change in lactate level/h & 0.60 & 0.98 & 100 & 71.2 & 38.2 & 100 \\
\hline \multicolumn{7}{|l|}{ Single-ventricle disease $(n=43)$} \\
\hline Initial lactate level & 5 & 0.83 & 100 & 48.6 & 30.8 & 100 \\
\hline Maximal change in lactate level $/ \mathrm{h}$ & 0.60 & 0.99 & 100 & 51.4 & 32 & 100 \\
\hline \multicolumn{7}{|l|}{ Two-ventricle disease $(n=43)$} \\
\hline Initial lactate level & 5 & 0.44 & 40 & 73.7 & 16.7 & 90.3 \\
\hline Maximal change in lactate level/h & 0.60 & 0.99 & 100 & 89.5 & 55.6 & 100 \\
\hline
\end{tabular}

$A U C$, Area under the curve; $P P V$, positive predictive value; $N P V$, negative predictive value.

PPV 32\%, and NPV 98\%. In subjects with 2-ventricle physiology, an initial lactate cutoff value of $5 \mathrm{mmol} / \mathrm{L}$ had an AUC of 0.80 , PPV 31\%, and NPV 97\%. In 2-ventricle subjects, a lactate increase cutoff value of $0.6 \mathrm{mmol} / \mathrm{L} / \mathrm{h}$ had an AUC 0.91, PPV 36\%, and NPV 99\%.

The predictive performance of the lactate values in the subgroup of neonatal patients (age $<30$ days) both overall and stratified by single- versus 2 -ventricle physiology is summarized in Table 3. For all neonates, an increase in lactate of $0.6 \mathrm{mmol} / \mathrm{L} / \mathrm{h}$ had near-perfect discrimination between outcomes (AUC, 0.99). There was again a relatively low PPV and high NPV for both the initial lactate and increase in lactate level. The notable exception was a PPV of $56 \%$ for an increasing lactate level in the 2-ventricle, neonatal cohort.

Similar to the other analyses, in groups stratified by RACHS-1 score, the AUCs indicated good discriminatory ability for the increase in lactate for both low and high RACHS-1 subgroups and high NPV and limited PPV. In subjects with a RACHS-1 score 1 to 4 , an initial lactate cutoff value of $5 \mathrm{mmol} / \mathrm{L}$ had an AUC of 0.82 , PPV $43 \%$, and NPV $98 \%$. Those with a RACHS-1 score of 5 to 6 had an AUC of 0.91 , PPV of $31 \%$, and NPV of $99 \%$. In subjects with a RACHS-1 score of 1 to 4 , a lactate increase of 0.6 $\mathrm{mmol} / \mathrm{L} / \mathrm{h}$ had an AUC of 0.78 , PPV of $24 \%$, and NPV of $99 \%$. Those with a RACHS- 1 score of 5 to 6 had an AUC of 0.85 , PPV of $32 \%$, and NPV of $95 \%$. Additionally, we performed a separate analysis stratifying by the operative use of circulatory arrest. The results of that analysis were nearly identical to the analysis stratified by the RACHS-1 score, likely reflecting the large majority of the infants requiring circulatory arrest who had a RACHS-1 score of 5 or 6 .

\section{Secondary Outcomes}

A total of 33 patients required an unplanned cardiac reoperation, with 16 of these occurring in patients who had demonstrated an increase in lactate $>0.6 \mathrm{mmol} / \mathrm{L} / \mathrm{h}$. An increasing lactate level increased the odds of an unplanned reoperation by 4.5 (95\% CI, 2.1-9.9; $P<.0001)$.

When stratified by lactate values, the median duration of mechanical ventilation, total ICU stay, and total hospital stay were significantly longer in both the high initial lactate and the increasing lactate groups compared with the low lactate groups. For subjects with an initial lactate level of $\geq 5 \mathrm{mmol} / \mathrm{L}$ compared with $<5 \mathrm{mmol} / \mathrm{L}$, the mechanical ventilator duration was a median of 5 days (IQR, 4-11) versus 3 days (IQR, 2-6; $P<.0001$ ), the total ICU stay was a median of 11 days (IQR, 6-18) versus 6 days (IQR, $3-10 ; P<.0001)$, and the total hospital stay was a median of 21 days (IQR, 16-38) versus 11 days (IQR, 7-18.5; $P<.0001)$. For subjects with a lactate increase of $\geq 0.6 \mathrm{mmol} / \mathrm{L} / \mathrm{h}$ compared with $<0.6 \mathrm{mmol} / \mathrm{L} / \mathrm{h}$, the mechanical ventilator duration was a median of 6.5 days (IQR, 5-11) versus 3 days (IQR, 2-5; $P<.0001$ ), the total ICU stay was a median of 12.5 days (IQR, 7-18) versus 5 days (IQR, 3-10; $P<.0001$ ), and the total hospital stay was a median of 22.5 (IQR, 16-36) versus 11 days (IQR, 7-17; $P<.0001$ ).

\section{DISCUSSION}

The present study is the largest report of serial lactate levels in infants after heart surgery with CPB. Our results have indicated that increasing lactate levels are very good discriminators for the outcome in infants after heart surgery.

In the overall cohort and all subgroups analyzed, the absence of increasing lactate levels had a very high association with a good outcome (strong NPV). In the absence of rapidly increasing lactate levels, clinicians can be confident that their current treatment approach is likely adequate in preventing tissue ischemia and will be associated with good outcomes. In contrast, the presence of rapidly increasing lactate levels was only a modest predictor of a poor outcome in all groups. This low PPV was not particularly surprising, because a PPV is contingent on the prevalence of the outcome in the population, and poor outcomes have a relatively low prevalence after pediatric cardiac surgery. In addition, in our institution, we often attempt to intervene in patients with increasing lactate levels by introducing therapies aimed at improving systemic oxygen delivery. This clinical approach might have confounded the relationship between the increasing lactate levels and the composite poor outcome. However, 
despite this potentially mitigating confounder, the presence of increasing lactate levels still was found to have excellent discriminatory ability in differentiating between low-risk patients and high-risk patients in whom the clinical outcome might still be in the balance and difficult to predict. In this high-risk group, therapeutic interventions should be considered when increasing lactate levels are detected.

Neonates with 2-ventricle physiology were a notable exception to the relatively low PPV of increasing lactate levels, with a $56 \%$ PPV for a poor outcome. This significant finding should guide the clinician to re-evaluate potential therapeutic interventions to prevent poor outcomes in neonates with 2-ventricle heart disease in whom lactate is increasing.

Several previous studies have considered the predictive ability of postoperative lactate levels using varying lactate measurements, including the initial lactate level, ${ }^{2-6,8,9}$ peak lactate level,,$^{2,3,6,13}$ and "lactime," a measurement of serum lactate clearance over time as evidenced by the total duration of lactate higher than a certain cutoff level, often $2 \mathrm{mmol} / \mathrm{L}$. $^{2,3}$ These studies examined lactate levels over varying postoperative periods from 24 to 72 hours. The initial lactate level might be indicative of the patient's immediate postoperative condition; however, the initial lactate level has often been found to have only modest predictive value, likely reflecting the physiologic changes that can occur in the immediate postoperative period. Our data have also demonstrated that the initial lactate level appears to be a reasonable predictor but was inferior to an increase in lactate in discriminating between groups. Peak lactate measurements have demonstrated greater predictive values than the initial lactate levels for a poor outcome. However, the peak lactate level is a suboptimal clinical marker, given that the maximal tissue oxygen debt has likely already occurred by the time of measurement and intervention might not prevent the resulting sequelae of tissue ischemia. Similarly, multiple studies have examined lactime. Although lactime has been shown to have good predictive value, similar to that of the peak lactate level, the optimal opportunity to prevent damage from tissue ischemia might have passed by the time the measurement has been obtained.

In $90 \%$ of our patients with a significant increase in serum lactate, the measured increase preceded the eventual resulting peak after the increase in serum lactate, with a median lead time of 4.6 hours. This interval could allow additional therapies to be initiated, targeting improved tissue oxygen delivery, before maximal tissue ischemia and its sequelae have occurred. Thus, lactate measurements that are indicative of peak tissue ischemia, such as peak lactate and lactime, might have good ability to predict the outcome, but they are less likely to allow effective intervention. An increasing lactate level might allow prevention of patient morbidity by allowing an early therapeutic response, making this a more desirable clinical marker.

Our result demonstrating an increased odds of unplanned cardiac reoperation with an increasing lactate level is also of interest. Unplanned reinterventions were recently demonstrated to have a strong association with increased mortality in neonates with CHD. ${ }^{16}$ Increasing lactate levels could prompt earlier evaluation and consideration for reoperation. This approach might decrease the period that an infant is exposed to inadequate tissue oxygenation and potentially preserve end-organ function after heart surgery.

Individual center practice patterns will vary in their response to lactate levels. Thus, each individual ICU that monitors serial lactate levels must determine which measured increase in lactate will best predict the outcome in their own center. Benchmarking the lactate levels and using them to improve outcomes has been demonstrated to be effective in improving the outcomes in other centers. ${ }^{17,18}$

\section{Study Limitations}

The present study had several limitations. First, as previously stated, lactate levels were routinely measured with every arterial blood gas draw in our CICU. Therefore, infants who appeared more critically ill likely had their lactate level measured more often than healthy-appearing postoperative infants, leading to a potential selection bias. Second, treating clinicians were not blinded to the lactate levels and might have factored the lactate measurements into their treatment decision-making. Third, this was a single-institution study performed at a large referral center, and these results might not be generalizable to other pediatric heart centers. Although we recognize these limitations, we believe that the large sample size of our study minimized some of these limitations and that a change in serial lactate levels remains a strong discriminator for the clinical outcome. Furthermore, because the treating clinicians had access to these measurements, our results actually demonstrated the "real world" predictive ability of lactate levels in CICU infants, and the results are no less compelling.

\section{CONCLUSIONS}

In infants after surgical repair of $\mathrm{CHD}$ using $\mathrm{CPB}$, an increase in lactate allows excellent discrimination between high- and low-risk groups for a poor outcome, has a high NPV, precedes post-peak tissue oxygen debt, potentially allowing for early therapeutic intervention, and allows serial monitoring to assess the treatment response. These factors make an increasing lactate level a clinically viable marker for the outcome and a potential trigger for therapeutic modification. Similarly, the absence of an increasing lactate level would be a reassuring finding. 
Infants with increasing lactate levels constitute a group at significant risk of morbidity and should prompt consideration of additional treatment options. Future multivariate studies to assess the interaction of lactate levels with other clinical markers are warranted to further improve our ability to predict the clinical outcomes in this patient population.

\section{References}

1. Weare JA, Gafford JT, Lu HS, Erdos EG. Purification of human kidney angiotensin I converting enzyme using reverse-immunoadsorption chromatography. Anal Biochem. 1982;123:310-9.

2. Ward PE, Sheridan MA, Hammon KJ, Erdos EG. Angiotensin I converting enzyme (kininase II) of the brush border of human and swine intestine. Biochem Pharmacol. 1980;29:1525-9.

3. Klauser RJ, Robinson CJ, Marinkovic DV, Erdos EG. Inhibition of human peptidyl dipeptidase (angiotensin I converting enzyme: kininase II) by human serum albumin and its fragments. Hypertension. 1979;1:281-6.

4. Klauser RJ, Robinson CJ, Erdos EG. Inhibition of kininase II (angiotensin I converting enzyme) by human serum albumin and its fragments. Adv Exp Med Biol. 1979;120B:477-85.

5. Johnson AR, Erdos EG. Metabolism of vasoactive peptides by human endothelial cells in culture: angiotensin I converting enzyme (kininase II) and angiotensinase. J Clin Invest. 1977;59:684-95.

6. Erdos EG. Angiotensin I converting enzyme. Circ Res. 1975;36:247-55.

7. Oshima G, Erdos EG. Inhibition of the angiotensin I converting enzyme of the lung by a peptide fragment of bradykinin. Experientia. 1974;30:733-4.

8. Erdos EG, Massion WH, Downs DR, Gecse A. Effect of the inhibition of angiotensin I converting enzyme in endotoxin and hemorrhagic shock. Proc Soc Exp Biol Med. 1974;145:948-51.
9. Igic R, Erdos EG, Yeh HS, Sorrells K, Nakajima T. Angiotensin I converting enzyme of the lung. Circ Res. 1972;31(Suppl 2):51-61.

10. Yang HY, Erdos EG, Levin Y. Characterization of a dipeptide hydrolase (kininase II: angiotensin I converting enzyme). J Pharmacol Exp Ther. 1971; 177:291-300.

11. Nasu K, Itoh H, Yuge A, Kawano Y, Narahara H. Insulin-like growth factor-I regulates vascular endothelial growth factor secretion by human oviductal epithelial cells and stromal fibroblasts. J Soc Gynecol Investig. 2006;13:368-71.

12. Nasu $K$, Itoh H, Yuge A, Nishida M, Kawano Y, Narahara H. Tumor necrosis factor-alpha regulates vascular endothelial growth factor secretion by human oviductal epithelial cells and stromal fibroblasts. Fertil Steril. 2007;87: 220-2.

13. Itoh H, Nasu K, Matsumoto H, Kawano Y, Yoshimatsu J, Narahara H. Hypoxia regulates vascular endothelial growth factor and soluble FMS-like tyrosine kinase-1 secretion by human oviductal epithelial cells and stromal fibroblasts. Fertil Steril. 2006;85:1097-102.

14. Chan KL, Ip P, Chiu CS, Cheung YF. Peritoneal dialysis after surgery for congenital heart disease in infants and young children. Ann Thorac Surg. 2003; 76:1443-9.

15. Ishikawa S, Takenaka K, Yanagihara K, Miyahara R, Kawano Y, Otake Y, et al. Matrix metalloproteinase-2 status in stromal fibroblasts, not in tumor cells, is a significant prognostic factor in non-small-cell lung cancer. Clin Cancer Res. 2004; 10:6579-85.

16. Tokudome T, Horio T, Yoshihara F, Suga S, Kawano Y, Kohno M, et al Direct effects of high glucose and insulin on protein synthesis in cultured cardiac myocytes and DNA and collagen synthesis in cardiac fibroblasts. Metab Clin Exp. 2004;53:710-5

17. Horio T, Maki T, Yoshihara F, Tokudome T, Suga SI, Kawano Y, et al. Gene expression, secretion, and autocrine effect of C-type natriuretic peptide in cultured adult rat cardiac fibroblasts. Circulation. 2001:104:307.

18. Horio T, Tokudome T, Maki T, Yoshihara F, Suga SI, Nishikimi T, et al. Gene expression, secretion, and autocrine action of C-type natriuretic peptide in cultured adult rat cardiac fibroblasts. Endocrinology. 2003;144:2279-84. 